\title{
Safe technique to treat occlusion of metallic endo- scopic ultrasound-guided hepaticogastrostomy stent using a novel basket catheter (with video)
}

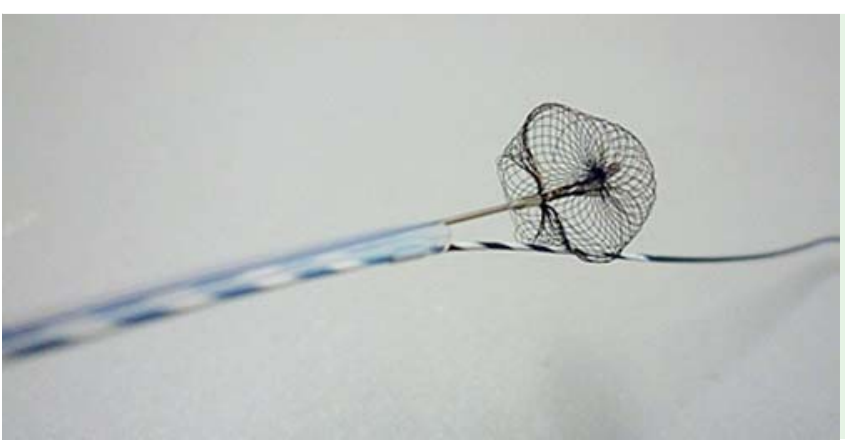

Fig. 1 Novel basket catheter for removal of biliary or pancreatic stones (Reforma; Piolax Medical Devices Inc., Kanagawa, Japan).

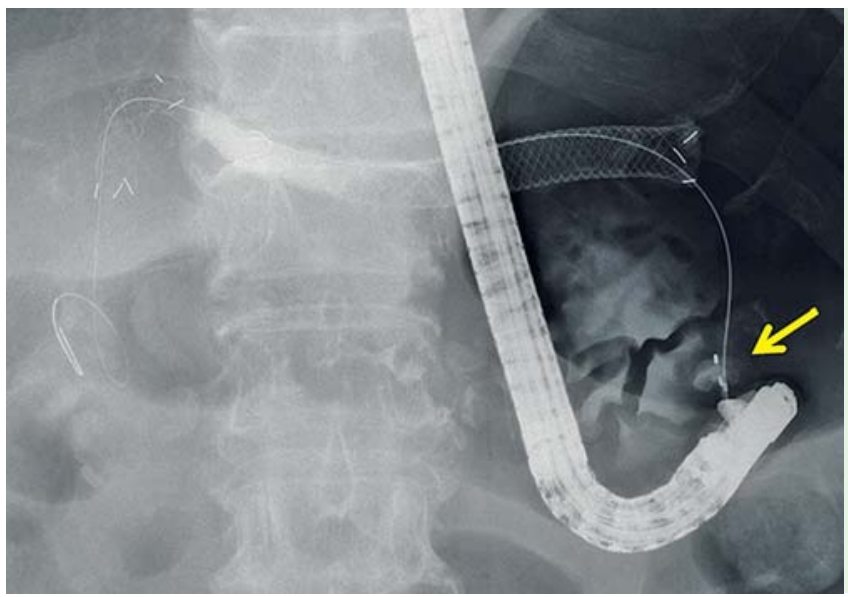

Fig. 3 We successfully cleaned the occluded EUS-HGS stent using a novel basket catheter (arrow).

Generally, to clean biliary metallic stents occluded by debris or food impaction, a balloon endoscopic retrograde cholangiopancreatography (ERCP) catheter has been widely used. Recently, a novel basket catheter has become available for removal of biliary or pancreatic stones (Reforma; Piolax Medical Devices Inc., Kanagawa, Japan) ( Fig. 1) [1]. This device may have clinical relevance not just for stone removal, but also for cleaning of biliary metallic stents.

If ERCP fails to resolve an obstructed biliary metallic stent, endoscopic ultrasound-guided hepaticogastrostomy (EUSHGS) is indicated [2]. If an EUS-HGS metallic stent is occluded by sludge, it is possible that the stent will migrate during pulling of the balloon catheter because of adhesion between the stent and the catheter. The novel basket catheter presented here is flexible and adjustable, so that if adhesion occurs between the stent and the catheter, the stent may still be safely cleaned.
We describe this safer method for cleaning an EUS-HGS metallic stent occluded by sludge using this novel basket catheter. The patient was a 76-year-old man who underwent EUS-HGS [3] because of obstructive jaundice caused by unresectable bile duct carcinoma and surgically altered anatomy. Nine months after the EUS-HGS he was admitted to our hospital, again because of obstructive jaundice. Endoscopic examination showed sludge in the EUSHGS metallic stent ( $\bullet$ Fig. 2 ), so we decided to clean the stent. To do this, we first inserted an ERCP catheter (MTW Endoskopie, Wesel, Germany) into the stent and advanced a 0.025 -inch guidewire (Revowave; Piolax) into the intestine, and injected contrast medium into the biliary tree. Next, we inserted a Reforma basket catheter into the metallic stent and pulled it into the stomach ( Fig.3, Fig.4). Once this procedure had been performed several times, the occluded stent was clear $(\bullet$ Video 1$)$.

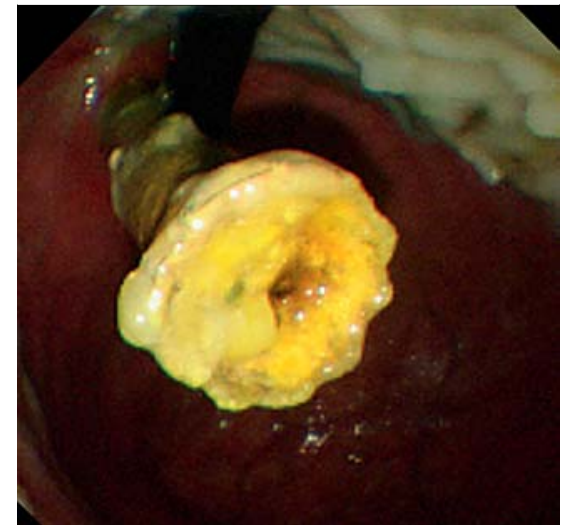

Fig. 2 Metallic stent occluded by sludge in a 76-year-old man 9 months after endoscopic ultrasound-guided hepaticogastrostomy (EUSHGS).
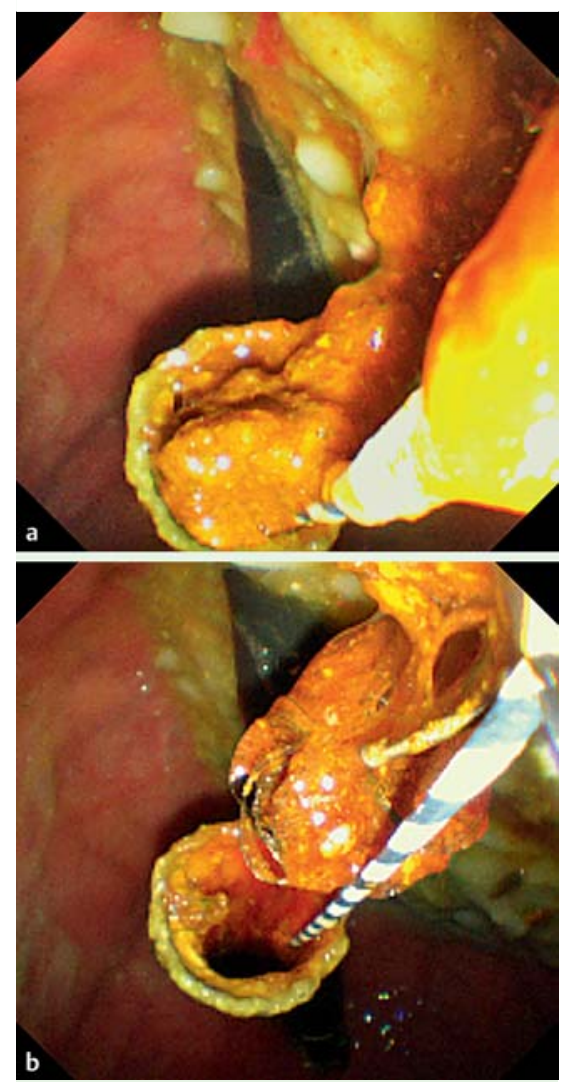

Fig. 4 a, b Sludge was successfully removed without any adverse events, including stent migration. 


\section{Video 1}

We advanced the endoscopic retrograde cholangiopancreatography catheter into the endoscopic ultrasound-guided hepaticogastrostomy stent, and injected contrast medium into the biliary tree. Next, we inserted the novel basket catheter and pulled it into the stomach. After we had performed this procedure several times, the occluded stent was clear.

Although additional cases will be required for validation, our method may have clinical relevance for cases of occluded biliary metallic stent such as the one presented here.

Endoscopy_UCTN_Code_TTT_1AR_2AZ

Competing interests: None
Takeshi Ogura, Akira Imoto,

Daisuke Masuda, Rieko Kamiyama, Saori Onda, Kazuhide Higuchi

Second Department of Internal Medicine, Osaka Medical College, Osaka, Japan

\section{References}

1 Sasahira N, Isayama $H$, Tokyokawa $Y$ et al. A novel basket catheter to facilitate enoscopic removal of pancreatic stones (with video). Gastrointest Endosc 2013; 78: 925 -929

2 Giovannini M, Dotti M, Boris E et al. Hepaticogastrostomy by echoendoscopy as a palliative treatment in a patient with metastatic biliary obstraction. Endoscopy 2003; 35 : 1076-1078

3 Ogura T, Masuda D, Imoto A et al. Endoscopic ultrasound-guided hepaticogastrostomy using two metal stents locked together. Endoscopy 2014; 46: E65-66
Bibliography

DOI http://dx.doi.org/ 10.1055/s-0034-1365435

Endoscopy 2014; 46: E234-E235

(C) Georg Thieme Verlag KG

Stuttgart · New York

ISSN 0013-726X

Corresponding author

Takeshi Ogura, MD, PhD

Second Department of Internal Medicine Osaka Medical College

2-7 Daigaku-machi

Takatsuki-shi

Osaka 569-8686

Japan

Fax: $+81-72-6846532$

oguratakeshi0411@yahoo.co.jp 